\title{
Antisense Oligonucleotides: Pharmacology and Delivery Strategies
}

\author{
Mohammed Abdul Samad*, Kavya Pandiri, Anjani Prasad Bojanapally \\ Department of Pharmacology, G. Pulla Reddy College of Pharmacy, Mehdipatnam, Hyderabad-500028, Telangana, India
}

\begin{abstract}
Antisense oligonucleotide therapy is a dominant drug discovery approach that can explicitly modify protein synthesis through numerous mechanisms. The limitations of antisense oligonucleotide (ASO) therapy in delivery strategies have been overcome in recent years with different ligands carriers, as well as, through nanocarriers. ASO therapy was successfully applied towards targeting a wide range of therapeutic areas. There is an expanding enthusiasm in extending the utilization of antisense compounds to numerous different diseases due to their safe and potential therapeutic outcomes. Thus, the present review attempted to elaborate on the fundamental idea of antisense technology, approaches, and safe and effective delivery methods.
\end{abstract}

Keywords: Antisense, Delivery, Mechanism, Oligonucleotide, Pharmacokinetics, Pharmacology.

\section{INTRODUCTION}

According to the recent designation, a synthetic singlestranded nucleic acid chain with 8 to 50 nucleotides is termed as an ASO. ${ }^{1}$ In 1978, Stephenson and Zamecnik first discovered the process of gene inhibition with ASO to stop viral replication in cell culture. ASO bounds through standard base pairing to their complement RNA, altering RNA function and thereby hamper the gene expression. They can be utilized to modify the synthesis of proteins and alter the synthesis of toxic proteins. ${ }^{2}$ The present review discussed the mechanism of ASO action, approaches, pharmacology, and various effective delivery methods.

\section{ANTISENSE APPROACH}

\subsection{Antisense Deoxyribonucleic Acid (ADA)}

Primary approach for ASO is utilizing single-stranded antisense deoxyribonucleic acids with 15 to 25 base pairs. They are intended to be correlative to discrete destinations on a particular targeted mRNA forming DNA:RNA hybrids. The mechanisms to restrain transcription process consist of directly binding to DNA, through formation of triple-stranded helix and combining with transcription factors. ${ }^{3}$

\subsection{Antisense Ribonucleic Acid (ARA)}

Another approach uses ribonucleic acids, which ties to a particular target mRNA, which obstructs the formation of a particular protein. This methodology requires acquaintance and production of a small part of the complete gene sequence. ${ }^{4}$

There are two general classes of ASOs utilized in antisense therapy. First is the single-stranded ASO that activates enzyme RNase $\mathrm{H}$, which causes degradation of the target RNA and another class is the doublestranded synthetic oligonucleotides that form RNAinduced silencing complex which then degrade the target RNA. ${ }^{5}$

\subsection{Advantages of ASOs}

- ASOs are a lot simpler to concentrate on than numerous different approaches to treat different diseases.

- Extent of sensitivity can be measured with ease and can be modified as needed.

- ASOs can be controlled and conveyed as needed without causing harm to healthy cells.

- They can be used in targeted drug therapies in place of conventional drugs with huge biodistribution and toxicities.

\section{Corresponding author}

Mohammed Abdul Samad

Email : mdabduls070@gmail.com; Tel.: +91-7702182242 
- ASOs are potential adjuvant in the treatments of neurological disorders, AIDS, cancer, diabetes, hepatitis, etc.

\subsection{Disadvantages of ASOs}

- Complex process and high cost of production.

- Intricacy in delivery into the body, especially through a pill as the human body has a characteristic barrier against it.

- Rapid excretion, deprivation by enzyme nucleases, and degradation by phagocytes in liver and spleen.

- Slow diffusion and binding in an extracellular fluid environment.

- Inevitable toxic effects because ASOs regulate both mutant and normal alleles.

- Challenge to determine the correct composition and dosage regimen to reduce unintended side effects caused by an alteration in genes.

\section{MECHANISM OF ASOS}

ASOs bring about the development of hetero-duplex ASO-mRNA complex, when combined and incorporated with the complement target mRNA that leads to activation of RNAse $\mathrm{H}$ enzyme or steric hindrance of ribosomal subunit binding. This mechanism causes debasement of ASO-mRNA complex and ultimately target protein knockdown. Oligonucleotide with RNase H enzyme activation, when targeted to any locale on the mRNA, causes its damage and debasement.

Further mechanisms of working of ASOs is by inward bounding to DNA/RNA directly into the nucleus and by altering the progress of mRNA, inhibition of 5'-cap formation, translation process, and activation of RNAse enzyme. $^{6}$

\section{SYNTHESIS AND DESIGN}

The initial phase in designing ASOs is recognizing the useful sequences for target inhibition, which should be a complement to that specific gene, and available for forming bound ASO complex. An ASO must be coordinated and designed in a structure that conveys adequately and contradicts deprivation in vivo. The affinity of ASOs to the target mRNA is a measure of the stability of the nucleic acid hybrid. The enhanced affinity of ASOs has a high probability of gene repressing action. ${ }^{7}$

\section{CHEMICAL MODIFICATIONS}

Synthetically designed unmodified ASOs have hostile and unfavorable characteristics that cause difficulties in their development. Degradation by nucleases, poor entry into cells, and low binding affinity for integral sequences are major hindrances. To overcome these obstructions, the backbones of oligonucleotides were chemically modified and arranged by the addition of phosphodiester bonds, ribose sugar bases, and nucleobases. ASOs are progressing due to increased chemical modification, which enhances their in vivo half-life, distribution throughout the body, potency, and decreases toxicity. ${ }^{8,9}$

\subsection{First Generation ASOs}

In these ASOs, sulfur is substituted in place of nonbridging phosphoryl oxygen of DNA. This substitution protects the ASOs from nuclease digestion and increases half-life with acceptable tissue distribution. Firstgeneration ASOs are potent and well-tolerated, typically with low toxicity.

\subsection{Second Generation ASOs}

These ASOs involves chemical modification of the ribose phosphodiester backbone linkage, or the heterocycle, or the sugar group. Substitution of 2'-position with an electronegative group, such as, 2'-O-methyl, 2'-O-methoxyethylgroup, or 2'-O,4'-C-methylene bridge gives an RNA, like C3'-endo conformation to the modified oligonucleotide that increases affinity and stability to a greater extent. These ASOs exhibit much improved pharmacokinetic characteristics.

\subsection{Third Generation ASOs}

Addition of peptide nucleic acids to chemically modify the ASOs guarantees further enhancements later on. An example is the phosphorodiamidate morpholino oligomers, which is designed with a morpholine ring in place of ribose ring that has phosphoroamidate linkages. ${ }^{10-13}$

\section{PHARMACOKINETICS}

\subsection{Route of Administration}

The primary route for systemic delivery of ASOs is by parenteral injection via subcutaneous (SC) injection or intravenous (IV) infusion.

\subsection{Absorption and Distribution}

On administration by subcutaneous injection, ASOs are quickly absorbed with highest plasma concentrations within 3 to 4 hours and rapidly declines by distribution phase, in which ASOs relocate from circulation to tissues 
in minutes or a few hours. The elimination phase then is much slower. Many biological hindrances remain between the oligonucleotide and its site of action. Modified ASOs broadly bound to the plasma proteins $(85 \%)$, facilitates uptake in tissues, increase tissue halflife, prevent loss of drug in renal excretion, and modulate protein binding. A deficit charged oligonucleotides bound inadequately to target mRNA, which further leads to its rapid excretion. ${ }^{14,15}$ Most ASOs are unable to cross the vital barriers, such as, blood-brain barrier and placenta. Nevertheless, the delivery in the cerebral spinal fluid may result in extensive distribution into spinal cord and brain tissue. ${ }^{16}$

\subsection{Metabolism and Clearance}

Compared with bolus administration, gradually IV infused ASOs brought about considerably more uptake in the liver, incriminating a saturated uptake process. Liver, kidney, lungs, bone marrow, spleen, lymph nodes, and adipocytes were reported to have the highest accumulated concentrations. ${ }^{4}$ The attached ASO influence the metabolism of the target RNA. ${ }^{17}$

\section{DELIVERY OF ASOS}

ASO do not readily permeate into the cell membranes due to the lipophilic lipid bilayer. Consequently, suitable chemically-based methodologies for delivery of ASO possibly will be supportive in ASO therapeutics. Endocytosis is a common process for cellular uptake of nucleotides. It depends on the cells the regular process of receptor interceded endocytosis. The downside of this strategy is the long time period and inefficient outcomes. ${ }^{18,19}$ The following are several strategies developed for the effective delivery of ASOs.

\subsection{Micro-Infection}

In this method, the ASO molecule is injected into the cell. This method is very reliable, but only about 100 cells can be infused in one day, since it requires the highest accuracy to inject a smaller molecule in very small cell. $^{20}$

\subsection{Liposome Encapsulation}

This method is the best strategy for huge amounts of ASOs in lipid vesicles, yet additionally expensive one. Liposome encapsulation can be accomplished by utilizing those substances which can cross phospholipids bilayer membrane of the cell. Subsequent liposome converges with the cell layer permitting the entrance of antisense into the cell. ${ }^{21,22}$

\subsection{Electroporation}

This is the conventional method of transferring an antisense nucleotide sequence into a cell. The antisense molecule is permitted to go through the cell film under the influence of a shock that is applied to the cells. ${ }^{23,24}$

\subsection{Antisense Polygalacturonase Gene}

The enzyme polygalacturonase is concerned with the breakdown of pectin, a component of cell walls. To slow the softening process, the Flavr Savr utilizes antisense technology to block polygalacturonase enzyme production. The antisense polygalacturonase-RNA is complementary to the mRNA produced by normal polygalacturonase genes, which will restrain the polygalacturonase enzyme by disabling their mRNA. This partial double-stranded complex is also rapidly degraded. ${ }^{25}$

\subsection{Polycationic Cell-Penetrating Peptides (PCPP)}

These PCCP primarily bind to glycosaminoglycans present on cell surface, followed by endocytotic uptake, and which ultimately releases the loaded ASO to the targeted cytosol. Generally, PCPPs deliver oligonucleotides with uncharged peptide nucleic acids, morpholino ring structure, and disulfide linkages. ${ }^{26}$

\subsection{Cell Targeting Ligand (CTL)}

Upon administration, cell-targeting ligands attach to receptor on the surface of the cell with high affinity that is able to go through proficient internalization. A large range of antibodies and polypeptides as potential ligands are available. Delivery of ASOs using different cell-penetrating peptides achieved strong biological impacts at very small concentrations. ${ }^{27}$

\subsection{Nanocarriers for Oligonucleotide Delivery}

ASOs, when delivered with nanoparticle encapsulation, additionally enhance their targeted site of action and improve gene silencing activity. Nanoparticlebased delivery systems to deliver ASOs drugs are achieved by using various polymers, such as, polymeric nanoparticles, ${ }^{28}$ polymer micelles, ${ }^{29}$ quantum dots, ${ }^{30}$ and lipoplexes. ${ }^{31}$

\subsection{Cationic Polymer Complexes}

These cationic amphiphiles containing cholic acid moieties are linked via alkyl-amino side chains. The 
cholic acid moieties allow entry through the lipid bilayer, while cationic alkyl-amino chains assist ASOs for binding to its receptor or target site. These amphiphile provides a huge enhancement in targeting the ASO. ${ }^{32}$

\subsection{Viral Vectors}

Antisense sequences encoding into viral vectors with small nuclear RNAs have been built for permitting proper subcellular localization of ASO with pre-mRNAs. Viral vectors, such as, retroviral, adenoviral vectors, dominate clinical gene therapy which addressed excellent delivery approach for ASOs. ${ }^{33,34}$

\subsection{Polycations}

Polycation is a well-known methodology intracellular delivery of therapeutic drugs, which includes the utilization of polyethylene amine complexes and dendrimers. They apply proton-sponge effect, by which the content of endosomes is released into the surrounding. ${ }^{35,36}$

\subsection{PEGlyated Polycations}

PEGylated compounds have been developed to sterically protect the delivery vehicles from the undesired effects of blood components. It also overcomes the impacts of serum protein binding and hence, prevents the adverse effects. But, it might also decrease the uptake of ASOs by target cells. ${ }^{37}$

\subsection{Lipoplexes}

Lipoplexes consist of cationic lipids that interact with the anionic lipids of endosome membrane and exert destabilizing effects. This leads to the formation of nonbilayer structures and endosome instability. A study revealed that ASO conveyed by lipoplexes enter the cells by membrane fusion, which represents the functional induction of the target gene. ${ }^{38}$

\subsection{Bacterial Mini Cells}

Recombinant mini cells are being utilized as a targeted drug delivery system. Bacterial mini cells are sphereshaped cell, which lacks chromosomal DNA and are, thus, unable to proliferate. These mini cells, containing miRNA, siRNA, or plasmid are targeted for delivery to specific cells or tissues via surface expressing antibodies. ${ }^{39}$

\section{FUTURE PERSPECTIVE}

In the course of recent decades, ASO innovation has risen as a substantial way to deal with physiologic and pathologic procedures. Continuous in vitro examinations utilizing ASOs grant the portrayal of new targets and new therapeutic compounds. The delivery and stability issues are right now being effectively addressed, and it is soon likely to turn into a pillar of treatment, revealing insight into approaches to building helpful adequacy and specificity.

\section{CONCLUSION}

Regardless of the present use of ASOs is advancing; still, there are critical issues in utilizing these nucleic acid chains. Development of modified ASOs can now reach specific targeted tissues, such as, brain, placenta, liver, and spleen, and even in gaps in the endothelium, giving hopes for treating various illnesses of humans. Recently, ASOs as therapeutic agents have developed as a substantial approach to deal with specifically modulate gene expression, which has provoked an incredible covenant interest. Lipid and polymer-based nanocarriers still remain significant tools for the delivery of oligonucleotides. A range of ingenious methodologies has dealt with the challenges raised in oligonucleotide delivery. It appears to be likely that huge numbers of the issues in the oligonucleotide therapeutics field can be managed by sensible use of promising delivery strategies that right now exist in developed structures and that are rapidly emerging.

\section{REFERENCES}

1. Evers mm, Toonen LJ and Roon-Mom WMV. Antisense oligonucleotides in therapy for neurodegenerative disorders. Advanced Drug Delivery Reviews. 2015;87:90-103.

2. Scoles DR, minikel EV and Pulst SM. Antisense oligonucleotides. Neurology Genetics. 2019;5(2).

3. Fusco DD, Dinallo V, Marafini I, Figliuzzi mm, Romano B and Monteleone G. Antisense Oligonucleotide: Basic Concepts and Therapeutic Application in Inflammatory Bowel Disease. Frontiers in Pharmacology. 2019;10.

4. Geary RS, Wancewicz E, Matson J, Pearce M, Siwkowski A, Swayze E, Bennett CF. Effect of dose and plasma concentration on liver uptake and pharmacologic activity of a 2'-methoxyethyl modified chimeric antisense oligonucleotide targeting PTEN, Biochem Pharmacol. 2009;78(3):284-291.

5. Bennett CF, Swayze EE. RNA targeting therapeutics: molecular mechanisms of 309 antisense oligonucleotides as a therapeutic platform, Annu Rev Pharmacol Toxicol.2010;310(50):259-293.

6. Dias N, Stein CA. Antisense oligonucleotides: basic concepts and mechanisms. mol Cancer Ther.2002;1(5):347-55.

7. Shen L.et al. Evaluation of C-5 propynyl pyrimidinecontaining oligonucleotides in vitro and in vivo. Antisense Nucleic Acid Drug Dev. 2003;13:129-142.

8. Juliano RL. The delivery of therapeutic oligonucleotides. Nucleic Acids Res. 2016;44:6518-6548.

9. Crooke ST, Wang S, Vickers TA, Shen $\mathrm{W}$ and Liang XH. Cellular uptake and trafficking of antisense oligonucleotides. Nat. Biotechnol. 2017;35:230-237. 
10. Larsen HJ, Bentin T, Nielsen PE. Antisense properties of peptide nucleic acid. Biochim Biophys Acta. 1999;1489(1):15966.

11. Hoyer D, Zorrilla EP, Cottone P, Parylak S, Morelli M, Simola, N, Green R. Chemical Modifications of Antisense Oligonucleotides. Encyclopedia of Psychopharmacology. 2010;277-278.

12. Sharma VK and Watts JK. Oligonucleotide therapeutics: Chemistry, delivery and clinical progress. Future Medicinal Chemistry. 2015;7(16):2221-2242.

13. Shen $X$ and David RC. Chemistry, mechanism and clinical status of antisense oligonucleotides and duplex RNAs. Nucleic Acids Research: 2018;46(4):1584-1600.

14. Amantana A, Iversen PL. Pharmacokinetics and biodistribution of phosphorodiamidate morpholino antisense oligomers, Current Opinion in Pharmacology. 2005;5:550-555.

15. Iverson PL, Morpholinos in: S.T. Crooke (2007) Antisense Drug Technology: Principles, Strategies, and Applications, 2nd ed.CRC Press, Boca Raton, pp. 565-582.

16. Rigo F, Chun SJ, Norris DA, Hung G, Lee S, Matson J. et al. Pharmacology of a central nervous system delivered 2'-O-methoxyethyl-modified survival of motor neuron splicing oligonucleotide in mice and nonhuman primates, J Pharmacol Exp Ther. 2014;350(1):46-55.

17. Haley B, Zamore PD. Kinetic analysis of the RNAi enzyme complex, Nat Struct mol Biol. 2004;11(7):599-606.

18. Goyal N, and Narayanaswami P. Making sense of antisense oligonucleotides: A narrative review. Muscle and Nerve. 2017;57(3):356-370.

19. Ming, X. Cellular delivery of siRNA and antisense oligonucleotides via receptor-mediated endocytosis. Expert Opinion on Drug Delivery. 2011;8(4):435-449.

20. Xue X, Mao X, Zhou Y, Chen Z, Hu Y, Hou Z. et al. Advances in the delivery of antisense oligonucleotides for combating bacterial infectious diseases. Nanomedicine: Nanotechnology, Biology and Medicine. 2018;14(3):745-758.

21. Petrilli R, Eloy JO, Marchetti JM, Lopez RF, Lee RJ. Targeted lipid nanoparticles for antisense oligonucleotide delivery. Curr Pharm Biotechnol. 2014;15(9):847-855.

22. Sun $Y$, Zhao $Y$, Zhao X, Lee RJ, Teng L, and Zhou C. Enhancing the Therapeutic Delivery of Oligonucleotides by Chemical Modification and Nanoparticle Encapsulation. molecules (Basel, Switzerland). 2017;22(10):1724.

23. Damha Masad., Making Sense of Antisense. $2002<$ http:// www.erin.utoronto.ca/mbiotech/menu/damha.htm>

24. Joergensen M, Agerholm-Larsen B, Nielsen PE, Gehl J. Efficiency of cellular delivery of antisense peptide nucleic acid by electroporation depends on charge and electroporation geometry. Oligonucleotides. 2011;21(1):29-37.

25. Agrawal S. Kandimalla ER. Antisense therapeutics: is it as simple as complementary base recognition? mol Med Today. 2000;(6): 72-81.

26. Duchardt F, Fotin-MLeczek M, Schwarz H, Fischer R and Brock R. A comprehensive model for the cellular uptake of cationic cell-penetrating peptides. Traffic. 2007;8:848-866.
27. Holliger $\mathrm{P}$ and Hudson PJ. Engineered antibody fragments and the rise of single domains. Nat. Biotechnol. 2005;23:11261136.

28. Mayr J, Grijalvo S, Bachl J, Pons R, Eritja R, and Díaz DD Transfection of Antisense Oligonucleotides Mediated by Cationic Vesicles Based on Non-Ionic Surfactant and Polycations Bearing Quaternary Ammonium Moieties. International Journal of molecular Sciences. 2017;18(6):1139.

29. Vinogradov SV, Batrakova EV, Li S, and Kabanov AV. Mixed Polymer Micelles of Amphiphilic and Cationic Copolymers for Delivery of Antisense Oligonucleotides. Journal of Drug Targeting. 2004;12(8):517-526.

30. Cao X, Wang J, Deng W, Chen J, Wang Y, Zhou J et al. Photoluminescent Cationic Carbon Dots as efficient Non-Viral Delivery of Plasmid SOX9 and Chondrogenesis of Fibroblasts. Scientific reports. 2018;8(1):7057.

31. Wyrozumska P, Meissner J, Toporkiewicz M, Szarawarska M, Kuliczkowski K, Ugorski M, Walasek MA and Sikorski AF. Liposome-coated lipoplex-based carrier for antisense oligonucleotides. Cancer biology and therapy. 2015;16(1):66-76.

32. Nimesh S, Gupta N, Chandra R. Cationic polymer based nanocarriers for delivery of therapeutic nucleic acids. J Biomed Nanotechnol. 2011;7(4):504-520.

33. Imbert M, Dias-Florencio G, and Goyenvalle A. Viral VectorMediated Antisense Therapy for Genetic Diseases. Genes. 2017;8(2):51.

34. Yang L, Ma F, Liu F, Chen J, Zhao X and Xu Q. Efficient Delivery of Antisense Oligonucleotides Using Bioreducible Lipid Nanoparticles In vitro and In vivo. molecular TherapyNucleic Acids. 2020;19:1357-1367.

35. Xie J, Teng L, Yang Z, Zhou C, Liu Y, Yung BC, and Lee RJ. A Polyethylenimine-Linoleic Acid Conjugate for Antisense Oligonucleotide Delivery. BioMed Research International. 2013;1-7.

36. Mignani S, Shi X, Zablocka M and Majoral J. DendrimerEnabled Therapeutic Antisense Delivery Systems as Innovation in Medicine. Bioconjugate Chemistry. 2019;30(7):1938-1950.

37. Ikeda $\mathrm{Y}$ and Nagasaki Y. Impacts of PEGylation on the gene and oligonucleotide delivery system. Journal of Applied Polymer Science. 2013;131(9).

38. Ming X, Sato K and Juliano RL. Unconventional internalization mechanisms underlying functional delivery of antisense oligonucleotides via cationic lipoplexes and polyplexes. Journal of Controlled Release. 2011;153(1):83-92.

39. MacDiarmid JA, Mugridge NB, Weiss JC et al. Bacterially derived $400 \mathrm{~nm}$ particles for encapsulation and cancer cell targeting of chemotherapeutics. Cancer Cell. 2007;11(5):431445 .

How to cite this article: Samad MA, Pandiri K, Bojanapally AP. Antisense oligonucleotides: pharmacology and delivery strategies. Int. J. Appl. Pharm. Sci. Res. 2020;5(1): 7-11. doi: https://doi.org/10.21477/ijapsr.5.1.2

Source of Support: Nil

Conflict of Interest: None 